\title{
Regularity and Narrowness of the Intervals of the Microgrooves on the Rubbed Polymer Surfaces for Liquid Crystal Alignment
}

\author{
Takashi Ito, ${ }^{\dagger}$ Kazuo NaKanishi, Michinori NishiKawa, ${ }^{*}$ \\ Yasuaki YoKOYAMA, ${ }^{* *}$ and Yasumasa TAKEUCHI*** \\ Yokkaichi Research Center, Japan Synthetic Rubber Co., Ltd., \\ 100, Kawajiri-cho, Yokkaichi 510, Japan \\ * Tokyo Research Laboratory, Japan Synthetic Rubber Co., Ltd., \\ 3-5-1, Higashiyurigaoka, Asao-Ku, Kawasaki 215, Japan \\ ** Technical Center, Japan Synthetic Rubber Co., Ltd., \\ 100, Kawajiri-cho, Yokkaichi 510, Japan \\ *** Research and Development Department, Japan Synthetic Rubber Co., Ltd., \\ 2-11-24, Tsukiji, Chuo-ku, Tokyo 104, Japan
}

(Received July 18, 1994)

\begin{abstract}
It has been proved that genuine microgrooves on the rubbed polymer surfaces for liquid crystal alignment have very regular and narrow intervals. The minimum mean interval, $0.03 \mu \mathrm{m}$, is observed by field-emission scanning electron microscope (FE-SEM) after staining the polyimide alignment layer with ruthenium tetroxide $\left(\mathrm{RuO}_{4}\right)$ at room temperature. Its coefficient of variance is $14.7 \%$. Results on Atomic Force Microscope (AFM) measurements essentially coincide with that of FE-SEM. On the other hand, microgrooves of the poly(amic acid) which has poor ability to let the liquid crystals align, have less regular and wider intervals than polyimide. Five other polymers were also observed in the same manner. Non-destructive staining at room temperature and the existence of heavy metal compound on and just beneath the surface may make possible observation of the real topography of the rubbed polyimide surface. It is also emphasized from dichroic ratio measurements that observation without any distortion of the samples is very important.
\end{abstract}

KEY WORDS Polyimide / Poly(amic acid) / Liquid Crystal / Microgrooves / Rubbed Polymer Surfaces / Liquid Crystal Alignment Layer / Scanning Electron Microscopy/ Staining /

At present, most alignment layers of liquid crystal displays are made by rubbing polyimide coating on the indium-tin-oxide (ITO) substrate with nylon, or rayon cloth. The mechanism of rubbing which is still vague should be well studied and understood, because it is desirable to get alignment layer surfaces without the rubbing process.

The objective of this study is to make clear the mechanism of rubbing.

There are microgrooves on the rubbed surface of the liquid crystal alignment layer, ${ }^{1}$ but their intervals are too wide and too irregular to let the liquid crystals align as a monodomain. The polymer chain orientation of the alignment surface occurs by rubbing. ${ }^{2,3}$ Transfer and deposition of the fibers from the rubbing roll onto the surface sometimes occur, and these fibers sometimes become the cause of the alignment of the liquid crystals. ${ }^{4,5}$ These three facts have been said to be the origin of liquid crystal alignment, either individually or in combination. Besides these, other possible causes, for example, surface tension, excluded

† Present address: Tsukuba Research Laboratory, Japan Synthetic Rubber Co., Ltd., 25, Miyukigaoka, Tsukuba 305, Japan. 
volume effect, dispersion force, etc., are suggested. ${ }^{6}$

Since we could not decide which one was the principal cause of the alignment, we concentrated on microgrooves. We reviewed the results of observation on microgrooves in the literature. According to the conventional method, we could not obtain the real topography of the rubbed polyimide surface for liquid crystal alignment.

We found that after staining the rubbed polyimide surface with ruthenium tetroxide, $\mathrm{RuO}_{4}$, at room temperature, genuine microgrooves on the surface could be observed by scanning electron microscope (SEM). ${ }^{7}$ By this method, genuine microgrooves were found to have very regular and narrow intervals in the case of the polyimide in which good alignment of liquid crystals is possible.

On the other hand, microgrooves of the poly(amic acid) which has poor ability to let the liquid crystals align, have less regular and wider intervals than polyimide. Five other polymers were also observed in the same manner.

The dichroism of the liquid crystal cells made with these polymers as alignment layers was investigated by studying the orientation of azobenzene. From this investigation, heat distortion of microgrooves was examined.

\section{EXPERIMENTAL}

\section{Materials}

Polyimide (JIB : JSR OPTMER ${ }^{\circledR}$ AL1051) and poly(amic acid) (JPB) were obtained from Japan Synthetic Rubber Co., Ltd. Polystyrene (PST), poly(vinyl alcohol) (PVA), gelatin, poly(vinyl chloride) (PVC), and poly(methyl methacrylate) (PMMA) were obtained commercially. They were used as aligning polymers. The thickness on the ITO coated glass substrate was about 1000 angstrom(A). All the polymer layers were rubbed by a rayon cloth roll.

\section{Observation}

Polymer alignment layers were exposed to ruthenium tetroxide vapor at room temperature for 1 to $1200 \mathrm{~min}$. The alignment surfaces were observed by an electron probe X-ray microanalyzer (EPMA) and SEM after being coated with gold evaporated by sputtering or without gold layer. Field-emission scanning electron microscope (FE-SEM) and Atomic Force Microscope (AFM) measurements were carried out.

\section{Dichroism}

$4^{\prime}$-Nitro-4-dimethylaminoazobenzene $(1 \mathrm{wt} \%)$ in the liquid crystal (ZLI-1565) was used as a probe. Two sealing cells were used. One was heat sealing at $120^{\circ} \mathrm{C}$ and the other was UV curing. The dichroic ratio is defined as the ratio of the absorbance of azobenzene parallel $\left(A_{\|}\right)$and perpendicular $\left(A_{\perp}\right)$ to rubbing direction at the absorbance peak (around $470 \mathrm{~nm})$.

\section{RESULTS}

Observation by EPMA showed that there are many lines along the rubbing direction on the polyimide alignment surface (Figure 1). Intensity distribution of characteristic X-ray from $\mathrm{Ru}$ is localized. The mean interval $(X)$ and standard deviation $(\sigma)$ of the lines are $5.3 \mu \mathrm{m}$ and $1.02 \mu \mathrm{m}$, respectively. The coefficient of variance $(C V)$ is $19.2 \%$. The intervals of lines are fairly regular.

Many lines are observed on the polyimide alignment layer by SEM (Figures 2, 3). When the alignment layer is tilted at 65 degrees, lines become rows of hemispherelike deposits, or stackings of ruthenium compound on the microgrooves (Figure 4). As SEM has higher magnification and resolution than EPMA, smaller mean intervals, $0.57 \mu \mathrm{m}$ or $0.14 \mu \mathrm{m}$, are observed by SEM depending on the magnification, resolution and staining period. Standard deviation and $C V$ are $0.13 \mu \mathrm{m}$ and $0.02 \mu \mathrm{m}$, $22.8 \%$ and $14.3 \%$, respectively. The figures show 
T. Iто et al.

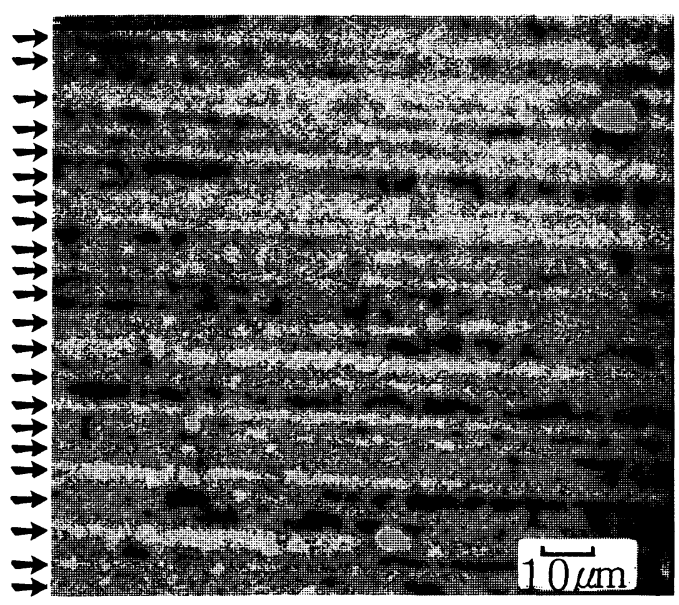

Figure 1. EPMA observation of the polyimide alignment surface. Arrows show lines of localization of ruthenium. Staining period, $60 \mathrm{~min} .(\times 2000)$

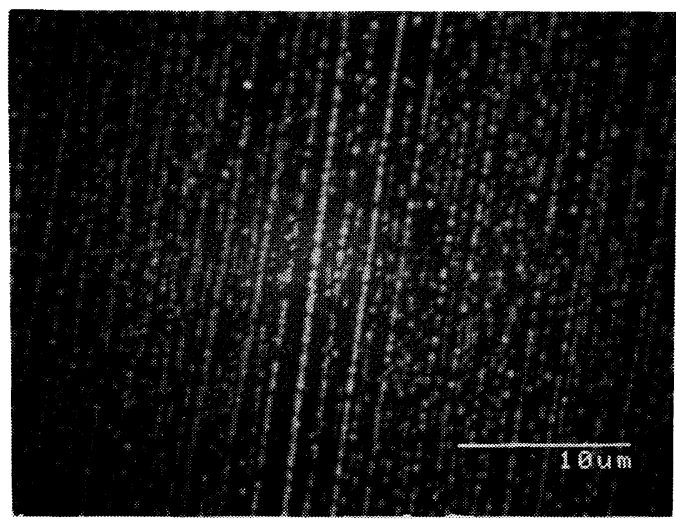

Figure 2. SEM micrograph of the polyimide alignment layer. Staining period, $60 \mathrm{~min} . X=0.57 \mu \mathrm{m}(\times 3000)$

that microgrooves lie at very regular intervals.

Narrow black lines with the mean interval of $0.03 \mu \mathrm{m}$ or $300 \mathrm{~A}$ are found on the alignment layer surface among big deposits of ruthenium by FE-SEM (Figure 5). A section of Figure 5 is enlarged for examination (Figure 6). These lines do not consist of rows of big hemispherelike deposits as observed by SEM. The standard deviation and $C V$ are $44 \mathrm{~A}$ and $14.7 \%$, respectively. This $300 \mathrm{~A}$ interval coincides with $200 \mathrm{~A}$, calculated from the free energy difference between the parallel and

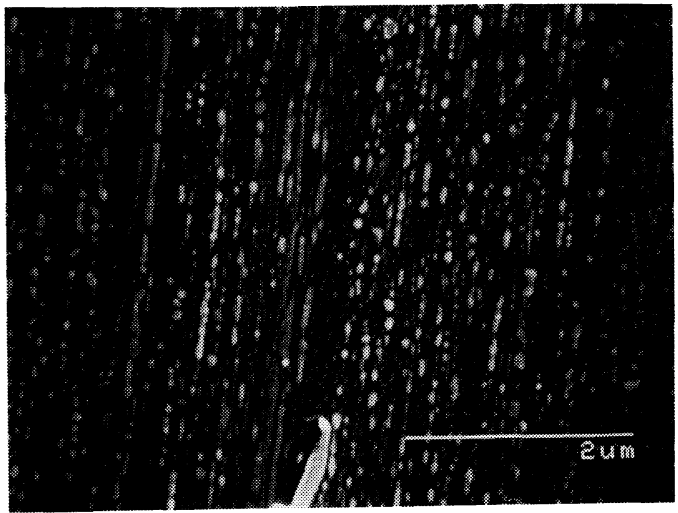

Figure 3. SEM micrograph of the polyimide alignment layer. Staining period, $10 \mathrm{~min} . X=0.14 \mu \mathrm{m}(\times 20000)$

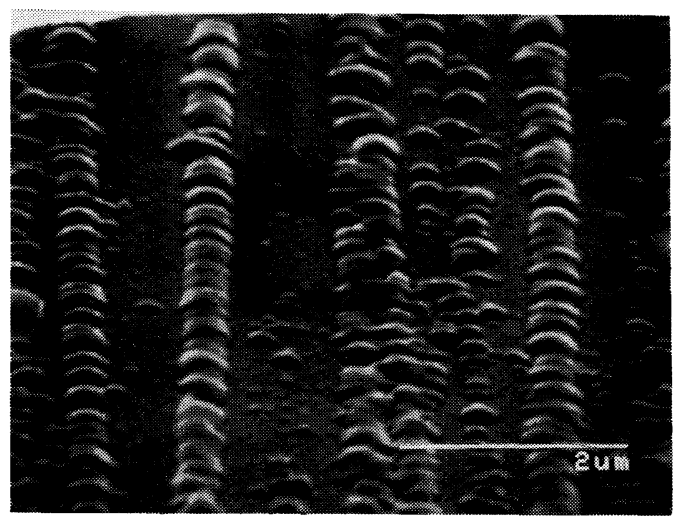

Figure 4. SEM micrograph of the polyimide alignment layer tilted at 65 degrees. Staining period, $60 \mathrm{~min} .(\times 20000)$

perpendicular alignment of the orientation vector of nematic liquid crystals on the microgrooves. $^{8}$

AFM measurements for another sample set other than those for SEM and FE-SEM are as follows (Table I).

The microgrooves on the poly(amic acid) layer which has poor ability to let liquid crystals align, have a mean interval of $1.81 \mu \mathrm{m}$, and $C V, 83 \%$ (Figure 7). These values are larger than the polyimide layer under the same conditions, $0.57 \mu \mathrm{m}$ and $22.8 \%$. This shows the importance of the regularity and narrowness of the intervals of the microgrooves on the liquid crystal alignment layers. Table II shows 


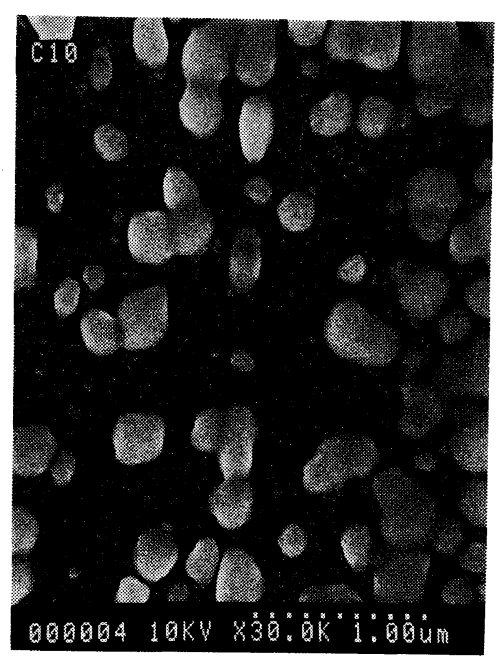

Figure 5. FE-SEM micrograph of the polyimide alignment layer. Staining period, $60 \mathrm{~min} . X=0.03 \mu \mathrm{m}$

$(\times 30000)$

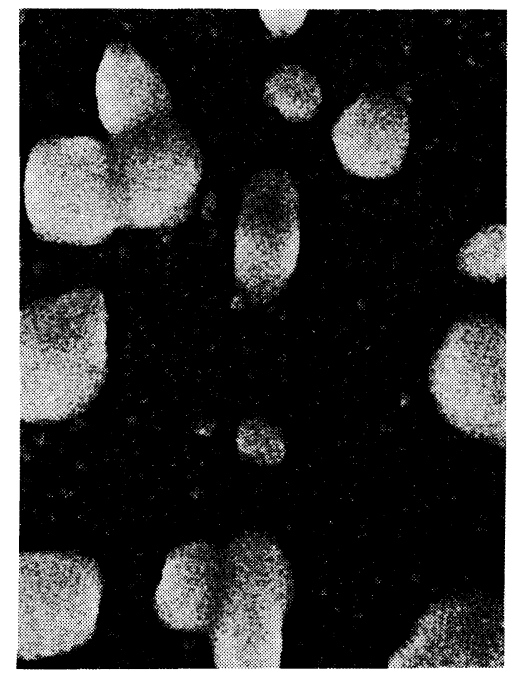

Figure 6. Enlarged section of Figure 5. microgroove intervals of polyimide and poly(amic acid).

Further investigation was carried out on layers of other polymers. Poly(vinyl chloride) (Figure 8) and poly(methyl methacrylate) (Figure 9) are not stained by $\mathrm{RuO}_{4}$. There appeared to be no structures or only faint lines on their surface.

Polystyrene, gelatin (Figures 10, 11), poly(vinyl alcohol) (Figure 12) are stained easily by $\mathrm{RuO}_{4}$. There are many deposits of ruthenium compounds on the surfaces. The deposits of ruthenium on the microgrooves of gelatin and poly(vinyl alcohol) make rows as on the polyimide and poly(amic acid). The intervals of gelatin among the microgrooves are much wider and much more irregular than polyimide. There seems to be no structural feature or regularity on the polystyrene layer surface.

The results of dichroism are summarized in Table III. Values in parentheses in Table III indicate $A_{\angle} / A_{\perp}$, where $A_{\angle}$ is the absorbance at

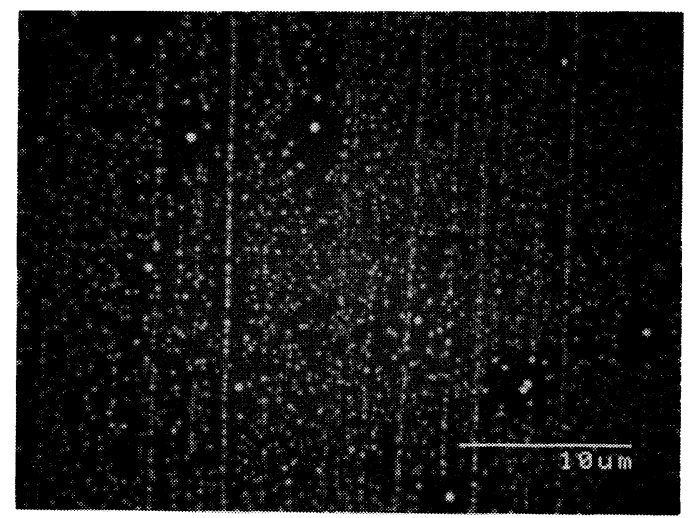

Figure 7. SEM micrograph of the poly(amic acid) layer. $(\times 3000)$

Table I. AFM measurement data

\begin{tabular}{|c|c|c|c|c|c|c|c|}
\hline & $\underset{\text { period }}{\mathrm{RuO}_{4} \text { staining }}$ & $X$ & $\sigma$ & $C V$ & Depth & $\sigma$ & $\mathrm{CV}$ \\
\hline & $\min$ & $\mathrm{nm}$ & $\mathrm{nm}$ & $\%$ & $\mathrm{~nm}$ & $\mathrm{~nm}$ & $\%$ \\
\hline JIB & 0 & 42 & 17 & 40 & 1.5 & 0.92 & 61 \\
\hline JIB & 10 & 57 & 20 & 35 & 1.1 & 0.49 & 43 \\
\hline
\end{tabular}


Table II. Microgroove intervals JSR polyimide

\begin{tabular}{ccccc}
\hline Apparatus & EPMA & SEM & SEM & FE-SEM \\
\hline $\begin{array}{c}\text { Staining } \\
\text { period }\end{array}$ & $60 \mathrm{~min}$ & $60 \mathrm{~min}$ & $10 \mathrm{~min}$ & $60 \mathrm{~min}$ \\
\hline$X=$ & $5.3 \mu \mathrm{m}$ & 0.57 & 0.14 & 0.030 \\
$n=$ & 21 & 56 & 6 & 8 \\
$\sigma=$ & $1.02 \mu \mathrm{m}$ & 0.13 & 0.02 & 0.0044 \\
$C V=$ & $19.2 \%$ & 22.8 & 14.3 & 14.7 \\
\hline $\begin{array}{c}\text { JSR poly(amic acid) } \\
\text { Apparatus }\end{array}$ & & & \\
\hline $\begin{array}{c}\text { Staining } \\
\text { Period }\end{array}$ & & SEM & & \\
\hline $\begin{array}{c}X= \\
n=\end{array}$ & & $60 \mathrm{~min}$ & \\
$\sigma=$ & & 1.81 & \\
$C V=$ & & 1.50 & \\
\hline
\end{tabular}

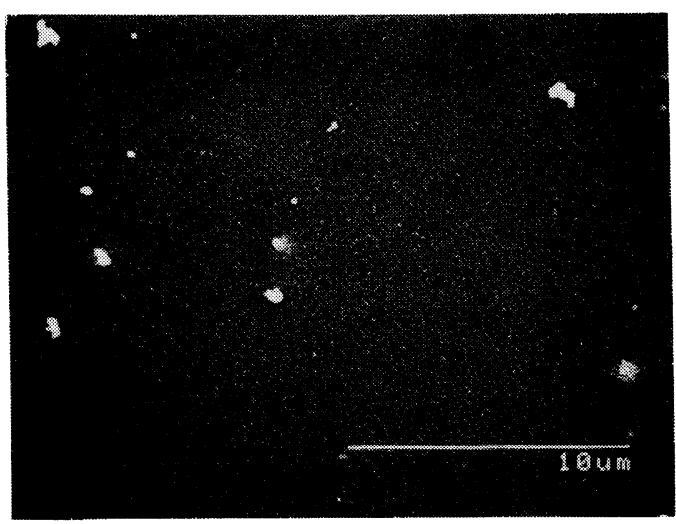

Figure 8. SEM micrograph of the poly(vinyl chloride) layer. Staining period, $60 \mathrm{~min} .(\times 5000)$

an angle of 45 degrees from the rubbing direction. The glass transition temperature $\left(T_{\mathrm{g}}\right)$ of each polymer is obtained from Differential Scanning Calorimetry (DSC) measurement. Molecular weight was not measured.

\section{DISCUSSION}

In the case of stainable polymers much

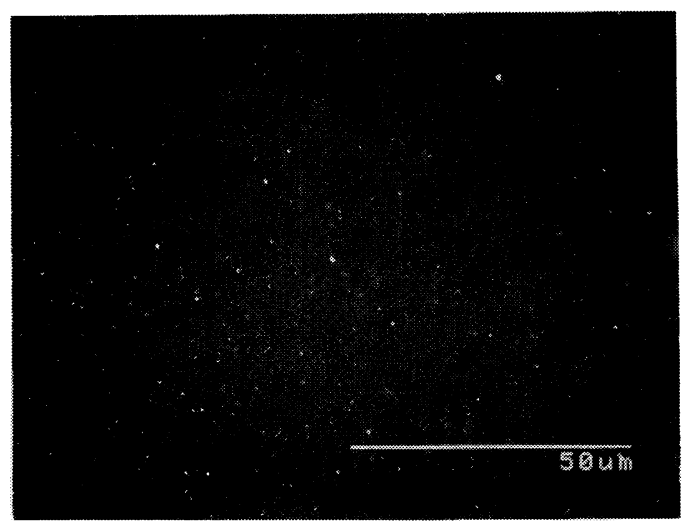

Figure 9. SEM micrograph of the poly(methyl methacrylate) layer. Staining period, $60 \mathrm{~min} .(\times 1000)$

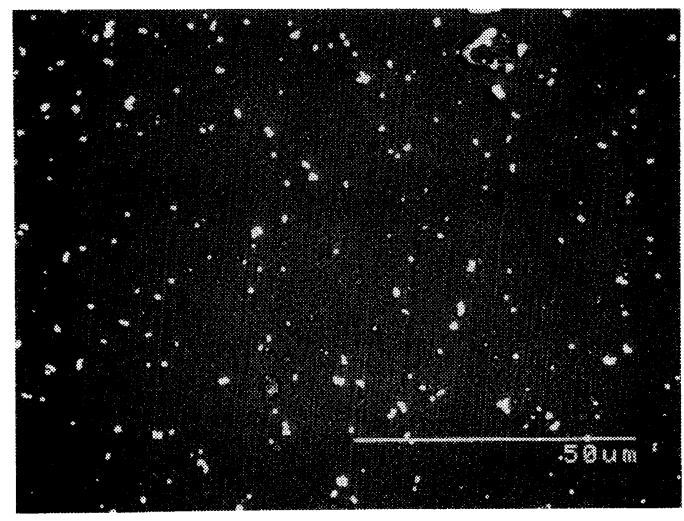

Figure 10. SEM micrograph of the layer of gelatin. Staining period, $60 \mathrm{~min} .(\times 1000)$

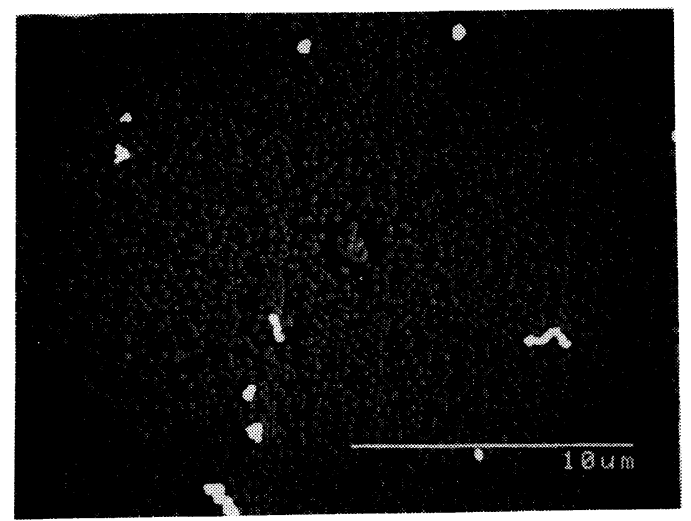

Figure 11. SEM micrograph of the layer of gelatin. Staining period, $60 \mathrm{~min} .(\times 5000)$ 


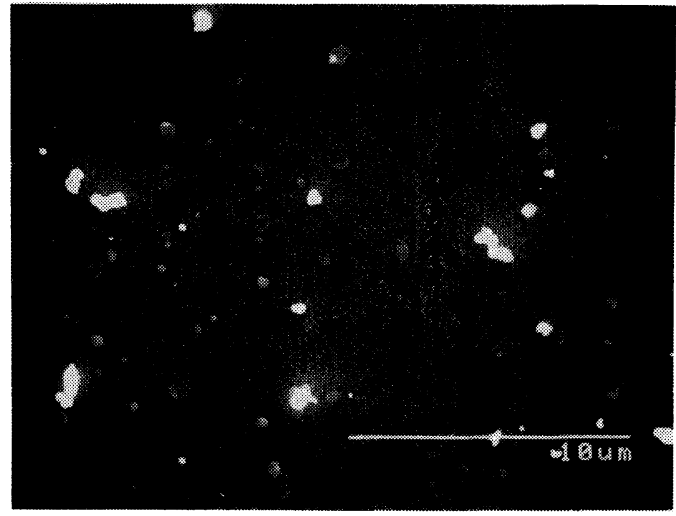

Figure 12. SEM micrograph of the layer of poly(vinyl alcohol). Staining period, $60 \mathrm{~min} .(\times 5000)$

Table III. Dichroic ratios of liquid crystal cells

\begin{tabular}{lccc}
\hline & & \multicolumn{2}{c}{$A_{\| /} / A_{\perp}\left(A_{\llcorner} / A_{\perp}\right)$} \\
\cline { 3 - 4 } Materials $^{\mathbf{a}}$ & $T_{\mathbf{g}} /{ }^{\circ} \mathrm{C}$ & $\begin{array}{c}\text { Heat sealing } \\
\text { cell }\end{array}$ & $\begin{array}{c}\text { UV curing } \\
\text { cell }\end{array}$ \\
\hline JIB & 380 & 6.2 & 7.1 \\
JPB & - & 6.1 & 5.0 \\
PVC & 74.5 & 1.5 & 3.9 \\
PMMA & 87.0 & 1.1 & 1.6 \\
Gelatin & - & $1.1(3.4)$ & $0.89(2.3)$ \\
PVA & 55.5 & 4.8 & 7.7 \\
PST & 42.5 & 4.0 & 6.3
\end{tabular}

a JIB, JSR OPTMER ${ }^{\circledR}$ AL1051; JPB, poly(amic acid); PVC, poly(vinyl chloride); PMMA, poly(methyl methacrylate); PVA, poly(vinyl alcohol); PST, polystyrene.

ruthenium diffuses into or is deposited on the layers depending on the period of staining. On the other hand, in the case of unstainable polymers, little ruthenium diffuses or is deposited during exposure to the vapor of $\mathrm{RuO}_{4}$.

Ruthenium deposits (as stackings) mainly on the microgrooves, particularly on wide microgrooves. These stackings might have some analogy with dewdrops on cold surfaces. Among the wide microgrooves, there are many narrow microgrooves whose surfaces are covered with ruthenium along the contour (Figure 13).

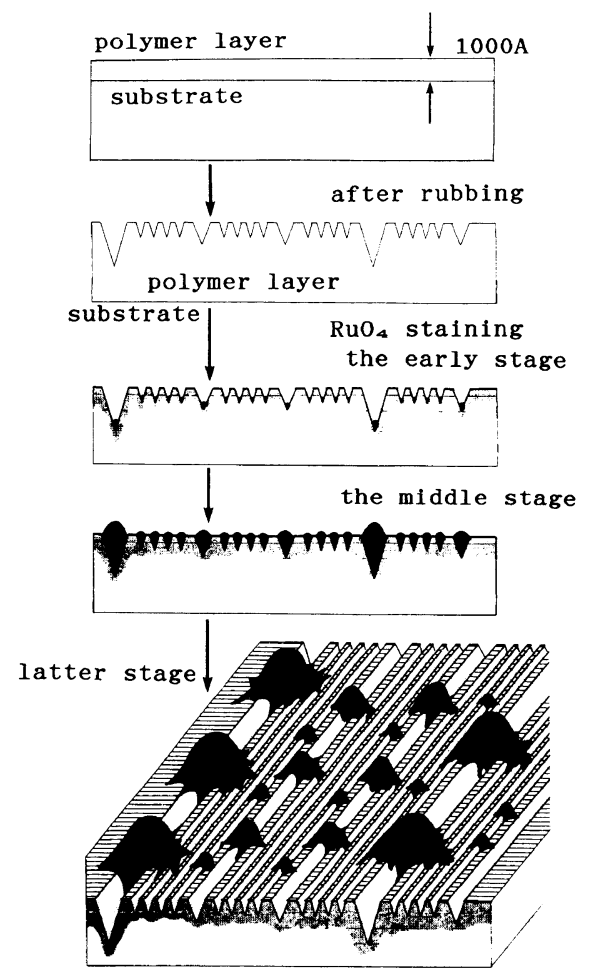

FIgure 13. Schematic drawings of staining $\mathrm{RuO}_{4}$.

It is well known that the image obtained from SEM is formed by the secondary electrons from the surface. And the generation of the secondary electrons depends on the topography, the electron density, the mean free path of the electrons, etc.

The non-destructive staining at room temperature and existence of heavy metal compound on and just beneath the surface may explain the real topography of the rubbed polyimide surface.

AFM measurements essentially coincide with values for FE-SEM. The experimental values of the interval and depth conform well to theoretical results. ${ }^{8}$ Based on depth dimension of the microgrooves, one can calculate the free energy difference at the alignment of liquid crystal molecules.

Because of the heat distortion of the polymer layers at the sealing cell, dichroic ratios were reduced to about $60 \%$ those of UV curing cell 
except gelatin, polyimide, and poly(amic acid). High glass transition temperature or thermosetting prevented decrease in these ratios.

\section{CONCLUSIONS}

Microgrooves are clearly observed at very narrow, regular intervals on a rubbed polyimide surface after staining with $\mathrm{RuO}_{4}$ at room temperature. This is not possible by the conventional method which destroys the surface profile by heat with the deposition of metal. It seems that not only the existence of microgrooves but also regularity and narrowness of the intervals are essential to the alignment of liquid crystals.

Acknowledgment. The authors express their appreciation to Miss Kiyoko Kawahara of the Tsukuba Research Laboratory in Japan Synthetic Rubber Co., Ltd. for AFM measurements.
This paper first appeared in the 1992 Society for Information Display International Symposium Digest of Technical Papers (SID 92 DIGEST), Volume XXIII, May 1992, p 393.

\section{REFERENCES}

1. S. Ishihara, H. Wakemoto, K. Nakajima, and Y. Matsuo, Liquid Crystals, 4, 669 (1989).

2. J. M. Geary, J. M. Goodby, A. R. Kmetz, and J. S. Patel, J. Appl. Phys., 62, 4100 (1987).

3. K. Nakajima, H. Wakemoto, S. Sato, F. Yokotani, S. Ishihara, and Y. Matsuo, Mol. Cryst. Liq. Cryst., 180B, 223 (1990).

4. J. A. Castellano, Mol. Cryst. Liq. Cryst., 94, 33 (1983).

5. M. E. Becker, R. A. Killan, B. B. Kosmowski, and D. A. Mlynski, Mol. Cryst. Liq. Cryst., 132, 167 (1986).

6. K. Okano and S. Kobayashi, "Ekishoh" (in Japanese), Baifu-kan, Tokyo, 1985.

7. T. Ito, K. Nakanishi, M. Nishikawa, Y. Yokoyama, and Y. Takeuchi, "Proceedings of 17th Liquid Crystal Symposium," Sapporo, Japan, 1991, 3F223.

8. J. Cognard, Mol. Cryst. Liq. Cryst., Suppl., 1, 1 (1982). 